\title{
Logarítmos: de sua invenção até a sala de aula.
}

\author{
Logarithms : his invention to the classroom
}

\author{
Saulo Portes dos Reis ${ }^{1}$ e Silvia Regina Vieira da Silva ${ }^{2}$ \\ ${ }^{1}$ Docente - Instituto Federal de São Paulo, Catanduva- SP- Brasil \\ spdreis@ifsp.edu.br \\ ${ }^{2}$ Docente - Departamento de Matemática da UNESP/Ilha Solteira ,UNESP- SP- Brasil \\ silviavieira_2004@yahoo.com.br
}

\begin{abstract}
Resumo
O presente trabalho apresenta uma proposta para o ensino do conceito de logaritmos a partir do contexto histórico de sua evolução. São abordadas duas perspectivas: a origem do logaritmos motivada pela necessidade de simplificar cálculos; a relação logarítmica presente no cálculo da área de uma faixa da hipérbole.

Nosso objetivo principal é deixar para o professor do ensino médio que, embora os logaritmos, hoje em dia, não sejam usados como "instrumento simplificador de cálculos", a compreensão desse conceito se faz importante por suas propriedades descobertas posteriormente a sua invenção.
\end{abstract}

Palavras-chave: Logarítmos. História da matemática. Napier. Quadratura da hipérbole.

\begin{abstract}
This paper presents a proposal for teaching the concept of logarithms from the historical context of its evolution. Two perspectives are addressed: the origin of logarithms motivated by the need to simplify calculations; the use of logarithmic relationship to calculate the area of a zone hypérbole.

Our main goal is that the teacher high school understand the logarithms, that although not calculations simplify (nowadays), understanding this concept becomes important by presenting others properties.
\end{abstract}

Keywords: Logarithmic. History of mathematics. Napier. Quadrature of hyperbole. 


\section{Introdução}

Este texto é um recorte de uma dissertação de mestrado profissionalizante que tratou o conteúdo de logarítmos. No referido trabalho optamos por organizar textos matemáticos associados a história da matemática, visando fornecer ao professor da rede básica de ensino uma abordagem diferenciada do conteúdo para o preparo de suas aulas.

Essa opção se deve a grande dificuldade apresentada pelos alunos na manipulação e compreensão desse conceito; acreditamos que a abordagem histórica possa facilitar a compreensão das propriedades dos logarítmos, ressaltando que as propriedades dos logarítmos foram a motivação inicial de sua "invenção"(termo utilizado por Napier). Portanto esse recorte não incluirá demonstrações extensas. Tais demonstrações se encontram em Reis (2014).

A grande maioria dos livros didáticos apresentam o conceito de Logaritmo como sendo o expoente de um número escrito em uma determinada base. Ou seja,

$$
\log _{a}(b)=x \Leftrightarrow b=a^{x},
$$

e $x$ é dito o logaritmo de $b$ na base $a$. A partir dessa definição são demonstradas as propriedades dos logaritmos.

Num primeiro momento essa abordagem parece ser a mais adequada, uma vez que fomos preparados a associar logarítmos com expoentes. Todavia essa escolha pode dificultar a compreensão das propriedades logarítmicas. Nossa experiência em sala de aula indica que a maior dificuldade do aluno está na compreensão (e consequentemente na utilização) das propriedades dos logaritmos:

$$
\begin{gathered}
\log _{a}(x \cdot y)=\log _{a}(x)+\log _{a}(y) \\
\log _{a}\left(\frac{x}{y}\right)=\log _{a}(x)-\log _{a}(y) \\
\log _{a} x^{b}=b \cdot \log _{a} x .
\end{gathered}
$$

Sendo assim, o presente trabalho tem por objetivo mostrar como se deu a construção e a evolução histórica dos logaritmos, bem como apresentar uma descrição matemática desse conceito, para que o professor tenha um maior embasamento teórico. Todos os resultados aqui apresentados se encontram nos livros pesquisados -Cajobi (2007); Eves (2010); Lima (2013); Lima (2012); Lima et al (2000); Maor (2012); Roque e Carvalho (2012); Stwart (2010)- apenas em alguns resultados indicaremos diretamente a fonte utilizada.

\section{Simplificando cálculos}

Poucas vezes na história da ciência uma nova ideia foi recebida com tanto entusiasmo e aceitação como foi com a invenção dos logaritmos. Uma ideia simples, porém muito prática, que economizou muito tempo dos cientistas da época (principalmente os astrônomos). Essa economia de tempo pode ter sido uma das razões que motivou a rápida aceitação e utilização desse novo conceito.

Os intelectuais dos séc XVI e XVII, em diversas áreas do conhecimento (astronomia, física, navegação,etc), anseavam pela realização de cálculos cada vez mais elaborados e consequentemente mais complexos e demorados. A demora na realização desses cálculos exigia muito tempo, o que impedia o desenvolvimento de outras teorias. Era preciso algum método de simplificar os cálculos, alguma forma de torná-los mais rápidos, e um dos métodos mais utilizados da época era a chamada prostaférese(esse termo vem do grego e significa somar e subtrair). Essa ideia consistia em usar tabelas de senos e cossenos para realizar operações de multiplicação e divisão com mais rapidez, uma vez que a partir das fórmulas do seno e cosseno da soma e subtração de arcos chegamos a:

$$
\operatorname{sen}(a+b)+\operatorname{sen}(a-b)=2 \cdot \operatorname{sen}(a) \cdot \cos (b) .
$$

Embora esse método pareça, a primeira vista, um pouco complicado, ele simplificava muito os cálculos. Essa simplificação reside no fato de que de um lado da equação temos uma adição e do outro lado uma multiplicação. Ora, é intuitivo que somar é um processo mais rápido do que multiplicar, por exemplo: somar 1432 com 16 exige 4 etapas, ao passo que multiplicar os mesmos números exige 12 etapas. Desse ponto de vista a prostaférese era muito útil e precisa. A diferença entre o resultado obtido pela calculadora e o resultado obtida pela prostaférese se deve a tabela de senos e cossenos utilizada. Como as tabelas de senos e cossenos utilizadas na época eram bem precisas (algumas com 14 casas decimais), os erros cometidos pela tilização da prostaférese não eram relevantes. No entanto, a prostaférese apresenta a limitação de não ser muito útil em agilizar multiplicação com 3 ou mais fatores e utilizála na divisão exige algumas iterações a mais do que a multiplicação. Diante desse contexto, se alguém criasse alguma técnica que simplificasse esses cálculos, economizaria muito tempo para os cientistas da época, que poderia ser utilizado em outras atividades mais criativas, descrever as órbitas dos planetas em torno do sol, por exemplo.

Encerramos essa seção com uma frase da pessoa que se propôs a encarar esse problema, e que apresentaremos na próxima seção: o matemático, físico, teólogo e astrônomo escocês: John Napier(1550-1617):

Já que não existe nada mais enfadonho, colegas matemáticos, na prática da arte matemática do que o grande atraso sofrido no tédio de extensas multiplicações e 
divisões, de encontrar razões, e na extração de raízes quadradas e cúbicas-e os muitos erros traiçoeiros que podem surgir: eu estive pensando, que arte segura eu poderia ser capaz de aperfeiçoar para tais mencionadas dificuldades. No final, após muito pensar, finalmente descobri uma surpreendente maneira de abreviar os procedimentos...e é uma tarefa prazerosa apresentar o método para o uso público dos matemáticos.(MAOR, 2012)

\section{Logaritmos de Napier}

John Napier era um nobre escocês que se preocupava mais com assuntos religiosos do que com matemática. Protestante e grande crítico da igreja católica, Napier defendia que o papa era o anti cristo e que o dia do juízo final estava entre 1688 e 1700. Essas ideias religiosas foram publicadas e ele tinha certeza que garantiria seu nome na história (pelo menos até 1700).

Porém, contrariando suas expectativas, sua fama não veio pelo seu ativismo religioso, ou por suas previsões catastróficas, mas pela invenção de uma técnica que minimizaria os esforços dos cientista de várias gerações, OS LOGARITMOS. Na época o conceito de logarítmo era entendido apenas como uma técnica de simplificação de cálculos (basicamente transformar multiplicação em adição) ${ }^{1}$.

A ideia de Napier era associar os termos de uma progressão geométrica (PG), aos termos de uma progressão aritmética (PA). A título de ilustração consideremos as sequências a seguir,

$$
\begin{gathered}
1,2,3,4,5,6, \ldots \\
q^{1}, q^{2}, q^{3}, q^{4}, q^{5}, q^{6}, \ldots
\end{gathered}
$$

notamos que os termos da primeira sequência formam uma PA de razão 1, enquanto que os termos da segunda sequência formam uma PG de razão $q$. Assim para o termo $n$ da PA, associamos o termo $q^{n}$ da PG.

Mas, como esse processo facilita operações?

Tomemos $q=2$. Se desejarmos multiplicar 16 por 32 , basta notar que $16=2^{4}$ e $32=2^{5}$. Assim $16 \cdot 32=$ $2^{4} \cdot 2^{5}=2^{4+5}=2^{9}$. Ora $2^{9}=512$, ou seja, $16 \cdot 32=512$. Então, para realizar a multiplicação $16 \cdot 32$ associamos a adição $4+5$ das potências desses números escritos na base 2. Assim, como no método da prostaférese, para resolver uma multiplicação (no caso $16 \cdot 32$ ) associamos uma adição $(4+5)$ para simplificar o cálculo. Com um abuso de linguagem, diremos que esses métodos "transformam"multiplicações em adições. No exemplo

\footnotetext{
${ }^{1}$ Atualmente o conceito é utilizado em várias áreas da ciência: cálculo de tempo de meia vida de substâncias radioativas; cálculo da concentração de hidrogênio $(\mathrm{pH})$; etc

${ }^{2}$ Vale ressaltar que a notação de potências $\left(q^{n}\right)$ ainda não era utilizada na época em que Napier criou os logarítmos
}

dado por $2^{9}=512$ Napier diria que 9 é o logarítmo de 512. Ressaltamos que Napier não utilizava o termo "base"pois adotara uma única base para todos os seus logarítmos, sendo assim o exemplo citado tem caráter meramente ilustrativo. Note que o logarítmo é o expoente da razão na PG (no caso exemplificado o expoente de 2), e o próprio termo tem essa denominação, pois logarítmo vem do grego: número da razão.

O próximo passo seria escolher um valor para $q$ de tal modo que um grande número de multiplicações pudessem ser facilitados. No exemplo citado utilizamos $q=2$, no entanto essa escolha não facilita cálculos de números que não sejam potências de 2 (3.47 por exemplo). Para isso seria necessário um número cujas potências crescessem lentamente, ou seja, um número bem próximo de 1. Após passar anos pensando no problema Napier escolheu o número 0,9999999 $=1-10^{-7}$.

Após a escolha da razão, o trabalho de Napier talvez tenha sido o menos empolgante da história da matemática, fazer inúmeros cálculos e obter uma tabela contendo uma quantidade de valores de logaritmos que simplificasse os cálculos. No entanto seu reconhecimento foi quase que imediato. Assim que surgiram as primeiras tabelas de logaritmos, os cientistas as utilizavam com frequência. Napier publicou seu trabalho em 1614 com o título "Uma descrição da maravilhosa regra dos logaritmos".

\section{Logarítmos Comuns}

Um dos que mais se entusiasmaram com a nova técnica de simplificar cálculos foi o primeiro professor de geometria do Gresham College, em Londres, Henry Briggs (1561-1630).

$\mathrm{O}$ fato dos logaritmos transformarem multiplicação em adição impressionou Briggs, em notação atual, $\log (x \cdot y)=\log (x)+\log (y) .{ }^{3}$ Todavia, os logaritmos de Napier apresentavam um incômodo: como a base utilizada era menor do que $1\left(1-10^{-7}=0,9999999\right)$, os logaritmos diminuíam a medida que os números aumentavam.

Briggs tentou solucionar o problema considerando uma base ligeiramente maior que 1. Para exemplificar vamos supor que Briggs tivesse adotado a base 1,00001, e determinar $\log (1,76)$ e $\log (17,6)$ nessa base. Utilizando uma calculadora, e a notação atual temos que $\log _{1,00001}(1,76)=56531,66$ e $\log _{1,00001}(17,6)=286791,32$.

Briggs notou que, embora $17,6=10 \cdot 1,76$, os seus respectivos logaritmos não apresentavam nenhuma relação. Isso acontecia por que $\log _{1,00001}(10)=230259,66$. Assim, se uma base como essa fosse adotada, a construção de uma nova tabela seria muito demorada. Briggs

\footnotetext{
${ }^{3}$ Alguns autores utilizam $\log (x)$ subebtendend base 10 , outros como Lima seubentendem base $e$, neste artigo explecitaremos a base em cada situação.
} 
então propôs que se adotasse $\log (10)=1$, pois assim, teríamos, $\log (10 \cdot x)=\log (10)+\log (x) \Rightarrow \log (10 \cdot x)=$ $1+\log (x)$.

A vantagem dessa convenção está no fato de que utilizamos base 10 em nosso sistema de numeração. Sendo assim, os logaritmos de 1,76 e 17,6 iriam diferir em apenas uma unidade. A título de ilustração, apresentamos os valores de $\log (1,76)$ e $\log (17,6)$ na base 10 .

$$
\begin{aligned}
& \log (1,76)=0,245512677 \\
& \log (17,6)=1,245512677
\end{aligned}
$$

Assim, se adotarmos um sistema de numeração em base 2 teremos $\log (2)=1$, generalizando a ideia de Briggs concluímos que a base de um sistema de logarítmos é o número $x$ que satisfaz $\log (x)=1$.

Note que essa convenção, nos leva a adotar $\log (1)=$ 0, pois, $\log (10)=\log (10 \cdot 1)=\log (10)+\log (1)$.

Briggs se encontrou com Napier em 1615. E após um longo período de admiração mútua ${ }^{4}$, Briggs apresentou suas ideias. Nasciam aí os chamados logaritmos briggsianos ou logaritmos comuns. Napier acatou as ideias de Briggs, porém, com uma idade avançada, não conseguiu construir uma nova tabela. Briggs se encarregou do trabalho. Em 1617 foi publicada uma tabela com os os logaritmos comuns de 1 a 1000, cada um com uma precisão de quatorze casas decimais. Em 1624, ampliou sua tabela calculando, também com quatorze casas decimais, os logaritmos de 1 a 20000 e de 90000 a 100000. Outros matemáticos se encarregaram de completar a tabela, porém com uma precisão de 10 casas decimais.

\section{A questão da base}

A maioria dos textos matemáticos didáticos (tanto do enino básico como superior) apresentam os logarítmos com expoentes de uma certa base. No entanto, como já foi mencionado, os logarítmos foram inventados antes que se usassem os expoentes. Dessa forma ressaltamos que os logarítmos como conhecemos hoje não são os mesmos de Naiper, todavia, as principais ideias propostas por ele ainda se mantém. Por exemplo, Briggs adotou $\log 10=1$, por utilizarmos base 10 em nosso sistema de numeração, mas qualquer número pode ser tomado como base, por isso aprendemos (e ensinamos) logarítmos sempre como sendo expoentes de uma base pré determinada. Se tomarmos uma base positiva qualquer ( $q$ por exemplo) podemos perceber que as ideias de Naiper residem na expressão $q^{a+b}=q^{a} \cdot q^{b}$. Assim ao adotarmos $q$ como base de um sistema de logarítmos teremos que $\log _{q}(q)=1$, uma vez que $q^{1}=q$.

\footnotetext{
${ }^{4}$ Conta-se que eles ficaram 15 minutos se olhando antes que a primeira palavra fosse dita
}

A partir dessas ideias podemos compreender outras propriedades de potências. Por exemplo tomando $q$ como um número positivo, $q^{a}=q^{a+0}=q^{a} \cdot q^{0}$. Sendo assim, devemos admitir $q^{0}=1$. Da mesma forma: $q^{0}=1=q^{a} \cdot q^{-a} q^{-a}=\frac{1}{q^{a}}$. Temos ainda $q=q^{1}=$ $q^{\frac{1}{2}+\frac{1}{2}}=q^{\frac{1}{2}} \cdot q^{\frac{1}{2}}\left(q^{\frac{1}{2}}\right)^{2}=q$. Ora o único número (positivo) que elevado ao quadrado vale $q$ é $\sqrt{q}$, assim, $q^{\frac{1}{2}}=\sqrt{q}$. Modificano ligeiramente o procedimento anterior teremos para qualquer número racional, $q^{\frac{a}{b}}=\sqrt[b]{q^{a}}$.

Ensinar logarítmos apenas como o expoente de um número em uma determinada base pode trazer o incômodo de não evidenciar por o número " $e$ ". Não é intuitivo que uma base irracional possa desempenhar um papel protagonista como o da base " $e$ ".

Passamos agora a estudar a "quadratura da hipérbole", um conteúdo que tem por objetivo tornar claro a importância do número"e"como base de um sistema de logarítmos.

\section{Fermat e as Quadraturas}

Quadratura refere-se ao ato de encontrar a área de uma figura a partir da área de figuras conhecidas. Por exemplo, encontramos a área de um trapézio retângulo decompondo-o em um triângulo e um retângulo. Dessa forma, é possível fazer a quadratura de qualquer polígono, uma vez que todo polígono pode ser "decomposto"em triângulos. Mas, e para fazer a quadratura de uma cônica? Daremos destaques aos estudos de Fermat.

Pierre de Fermat, um matemático francês nascido em 1601, teve contribuições significativas em diversas áreas da matemática, como a probabilidade, geometria analítica e teoria dos números. Por vezes Fermat é citado como o maior matemático francês do séc XVII. A presente seção tem por objetivo apresentar um dos problemas estudados por Fermat: a quadratura das curvas que, em notação atual, são escritas como $f(x)=x^{n}$, para $n \in \mathbb{Z}$.

Os métodos utilizados por Fermat se baseavam na ideia dos indivisíveis de Cavallieri ${ }^{5}$, uma ideia precursora do cálculo integral. A essência do método, consistia em escrever "a área abaixo de uma curva"como soma de retângulos cada vez menores. Em linguagem atual podemos afirmar que seria a soma de infinitos retângulos, com as medidas de suas bases tendendo a zero. Vamos mostrar como Fermat procedeu para encontrar a área desse tipo de curva.

Para $n \in \mathbb{N}$, as curvas são escritas como $f(x)=x^{n}$ são chamadas de parábolas generalizadas.

Primeiro vamos estabelecer que por "área abaixo da curva" $f(x)=x^{n}$, entendemos como a área limitada pela

\footnotetext{
${ }^{5}$ Esse método consiste em obter o volume de um sólido a partir da soma de infinitas porções cada vez menores
} 


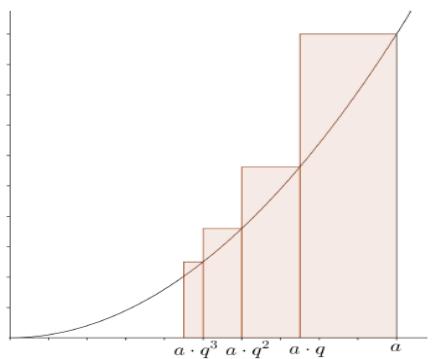

Figura 1: Quadratura das Parábolas Generalizadas.

curva, o eixo $y$ e a reta $x=a$.

Para calcular a área, Fermat primeiramente marcou pontos no eixo $x$ em progressão geométrica. Ou seja primeiro marcou o ponto $a$ e após adotou uma razão $q<1$ e marcou o ponto $q \cdot a$. Após os pontos $q^{2} \cdot a, q^{3} \cdot a$ e assim por diante. A figura 1 ilustra o procedimento adotado por Fermat.

O próximo passo consistiu em calcular a área de cada retângulo e somar todas as áreas obtidas. Descrevemos abaixo o procedimento.

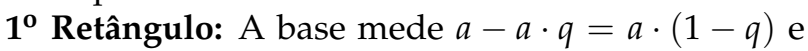
a altura mede $a^{n}$. Assim, denotando por $A_{1}$ a medida dessa área, temos:

$$
A_{1}=a \cdot(1-q) \cdot a^{n}=a^{n+1} \cdot(1-q) .
$$

$2^{\mathbf{o}}$ Retângulo: A base mede $a \cdot q-a \cdot q^{2}=a \cdot q \cdot(1-$ $q)$ e a altura mede $(a \cdot q)^{n}$. Denotando por $A_{2}$ a medida dessa área, temos:

$$
\begin{gathered}
A_{2}=a \cdot q \cdot(1-q) \cdot(a \cdot q)^{n}=\ldots \\
\ldots=a \cdot q \cdot(1-q) \cdot a^{n} \cdot q^{n}=a^{n+1} \cdot q^{n+1} \cdot(1-q) .
\end{gathered}
$$

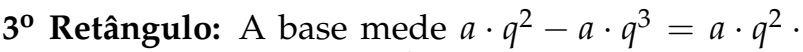
$(1-q)$ e a altura mede $\left(a \cdot q^{2}\right)^{n}$. Denotando por $A_{3}$ a medida dessa área, temos:

$$
\begin{gathered}
A_{3}=a \cdot q^{2} \cdot(1-q) \cdot\left(a \cdot q^{2}\right)^{n}=\ldots \\
\ldots=a \cdot q^{2} \cdot(1-q) \cdot a^{n} \cdot q^{2 n}=a^{n+1} \cdot q^{2 \cdot(n+1)} \cdot(1-q) .
\end{gathered}
$$

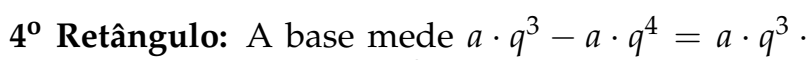
$(1-q)$ e a altura mede $\left(a \cdot q^{3}\right)^{n}$. Denotando por $A_{4}$ a medida dessa área, temos:

$$
\begin{gathered}
A_{4}=a \cdot q^{3} \cdot(1-q) \cdot\left(a \cdot q^{3}\right)^{n}=\ldots \\
\ldots=a \cdot q^{3} \cdot(1-q) \cdot a^{n} \cdot q^{3 n}=a^{n+1} \cdot q^{3 \cdot(n+1)} \cdot(1-q) .
\end{gathered}
$$

De um modo geral, temos:

i-ésimo Retângulo: A base mede $a \cdot q^{i-1}-a \cdot q^{i}=$ $a \cdot q^{i-1} \cdot(1-q)$ e a altura mede $\left(a \cdot q^{i-1}\right)^{n}$. Denotando por $A_{i}$ a medida dessa área, temos:

$$
A_{i}=a \cdot q^{i-1} \cdot(1-q) \cdot\left(a \cdot q^{i-1}\right)^{n}=\ldots
$$

$$
\begin{gathered}
\ldots=a \cdot q^{i-1} \cdot(1-q) \cdot a^{n} \cdot q^{(i-1) n}=\ldots \\
\ldots=a^{n+1} \cdot q^{(i-1) \cdot(n+1)} \cdot(1-q) .
\end{gathered}
$$

Assim a soma da área de todos retângulos seria:

$$
A_{\text {total }}=\sum_{i=1}^{\infty} A_{i}=\sum_{i=1}^{\infty} a^{n+1} \cdot q^{(i-1) \cdot(n+1)} \cdot(1-q) .
$$

Utilizando as propriedades de somatório, chegamos a:

$$
\begin{aligned}
& A_{\text {total }}=a^{n+1} \cdot(1-q) \sum_{i=1}^{\infty} q^{(i-1) \cdot(n+1)}=\ldots \\
& \ldots=a^{n+1} \cdot(1-q) \sum_{i=1}^{\infty}\left[q^{(n+1)}\right]^{i-1} .
\end{aligned}
$$

Seja $\lambda=q^{(n+1)}$, como $q<1$, então $\lambda<1$. Portanto o somatório

$$
\sum_{i=1}^{\infty} \lambda^{i-1}
$$

se reduz a soma dos infinitos termos de uma PG com razão menor do que 1 , ou seja,

$$
\sum_{i=1}^{\infty} \lambda^{i-1}=\frac{1}{1-\lambda}
$$

Temos então:

$$
A_{\text {total }}=a^{n+1} \cdot(1-q) \sum_{i=1}^{\infty}\left[q^{(n+1)}\right]^{i-1}=\frac{a^{n+1} \cdot(1-q)}{1-q^{(n+1)}}
$$

Mas, $1-q^{(n+1)}=(1-q) \cdot\left(q^{n}+q^{n-1}+q^{n-2}+\ldots+1\right)$, e portanto,

$$
\begin{gathered}
A_{\text {total }}=\frac{a^{n+1} \cdot(1-q)}{(1-q) \cdot\left(q^{n}+q^{n-1}+q^{n-2}+\ldots q^{1}+1\right)}=\ldots \\
\ldots=\frac{a^{n+1}}{q^{n}+q^{n-1}+q^{n-2}+\ldots q^{1}+1}
\end{gathered}
$$

Fermat percebeu que $A_{\text {total }}$ se aproximava da área da curva a medida que $q$ se aproximava de 1 . Se chamarmos a área abaixo da curva de $A_{c}$, na notação atual teremos,

$$
A_{c}=\lim _{i \rightarrow \infty} A_{\text {total }}=\frac{a^{n+1}}{n+1} .
$$

Um estudante de cálculo reconhece a expressão encontrada por Fermat, uma vez que,

$$
\int_{0}^{a} x^{n}=\left.\frac{x^{n+1}}{n+1}\right|_{0} ^{a}=\frac{a^{n+1}}{n+1} .
$$

Assim, Fermat encontrou de uma só vez uma expressão que permitia fazer a "quadratura"de uma família de curvas (no caso as parábolas generalizadas).

Uma outra classe de curvas estudadas por Fermat, foram as hipérboles generalizadas. São curvas do tipo 


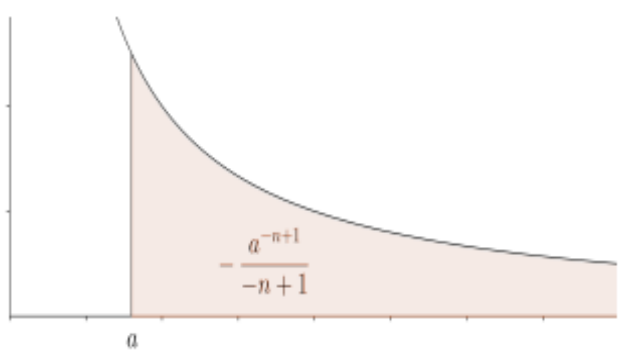

Figura 2: Área "abaixo"de uma Hipérbole generalizada".

$y=x^{-n}, n \in \mathbb{N}$. Essas curvas são diferentes das parábolas generalizadas, uma vez que seus valores funcionais vem do infinito e vão decrescendo sem nunca interceptar o eixo $x$. Com uma sutil modificação no método empregado para as parábolas generalizadas, Fermat concluiu que a fórmula $A_{c}=\frac{a^{n+1}}{n+1}(n \in \mathbb{N})$ também era válida para para as hipérboles generalizadas. No entanto duas ressalvas devem ser feitas: i- a área a ser determinada partia de um ponto $x=a$ e se estendia para o infinito; ii- o valor obtido após aplicar a equação é negativo, no entanto a área seria o módulo desse valor. Assim, se $n \in \mathbb{N}$, então a área limitada pelas retas $x=a, y=0 \mathrm{e}$ pela curva $y=x^{-n}$ seria dada por: $A_{c}=-\frac{a^{-n+1}}{-n+1}$.

Apesar de todo sucesso obtido pelas expressões de Fermat, havia um problema: a fórmula não era aplicável para a curva $y=x^{-1}=\frac{1}{x}$, uma vez que, se tentássemos aplicar os resultados de Fermat a essa curva obteríamos,

$$
A_{c}=-\frac{a^{-1+1}}{-1+1}=\frac{-1}{0} \text {. }
$$

Essa curva $\left(y=x^{-1}\right)$ é uma hipérbole, curva que Arquimedes não conseguiu fazer a quadratura. Resolver esse problema coube a um padre jesuíta belga contemporâneo de Fermat: Gregory Saint-Vincent.

\section{A quadratura da hipérbole e uma proprie- dade fundamental}

Gregory Saint-Vincent não é um nome muito conhecido entre os estudantes contemporâneos de matemática, sua principal atividade era tentar quadrar círculos. Ao tentar quadrar a hipérbole, Saint-Vicent percebeu que se construisse retângulos semelhantes aos usados por Fermat nas parábolas generalizadas, esses retângulos possuiriam áreas iguais. Vamos analisar esse fato.

Observando a figura 3 (fora de escala) ${ }^{6}$, vamos calcular as áreas dos retângulos apresentados.

Para a figura 3 vamos considerar que $a \cdot q^{4}$ seja o primeiro termo de uma PG de razão $\frac{1}{q}>1$ (uma vez

\footnotetext{
${ }^{6}$ Optamos por manter a figura dessa forma pois assim conseguimos uma melhor visualisação.
}

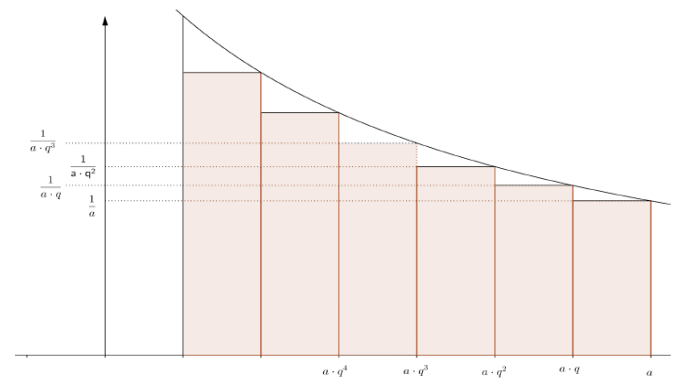

Figura 3: Quadratura da hipérbole.

que $q<1$ ). A esse termo associaremos o número 0 . Assim, o segundo termo da PG seria $a \cdot q^{3}$, ao qual associamos a área do $4^{\circ}$ retângulo. Ao próximo termo da PG associamos a soma das áreas do $3^{\circ}$ e $4^{\circ}$ retângulos, e ao próximo a soma das áreas do $2^{\circ}, 3^{\circ}$ e $4^{\circ}$ retângulo, e assim sucessivamente. Colocamos os resultados na tabela a seguir:

\begin{tabular}{|c|c|}
\hline PG & Áreas associadas \\
\hline$a \cdot q^{4}$ & 0 \\
\hline$a \cdot q^{3}$ & $1-q$ \\
\hline$a \cdot q^{2}$ & $2 \cdot(1-q)$ \\
\hline$a \cdot q$ & $3 \cdot(1-q)$ \\
\hline$a$ & $4 \cdot(1-q)$ \\
\hline
\end{tabular}

Portanto, partindo de um ponto de referência (no nosso caso $a \cdot q^{4}$ ) a distância a esse ponto cresce em PG, enquanto que as áreas associadas crescem em PA. Ou seja, existe uma relação logarítmica, uma adição (PA) associada a uma multiplicação (PG), entre as grandezas já citadas. Quem descreveu essa relação explicitamente foi um aluno de Saint Vincent, Alfonso de Sarasa. Essa foi a primeira descrição do uso do logaritmo como função, pois até então os logaritmos só eram utilizados como ferramentas de cálculo.

Denotaremos por $A(a, b)$ a área correspondida entre as retas $x=a, x=b, y=0$ e o gráfico da função $f(x)=\frac{1}{x}$ (vide figura 4). Uma propriedade de extrema relevância observada por Saint Vincent era que, na construção de retângulos para fazer aproximação das áreas, retângulos de bases proporcionais possuíam áreas iguais, ou seja, $A(a, b)=A(k \cdot a, k \cdot b)\left(k \in \mathbb{R}^{+}\right)$. A demonstração formal desse resultado foge aos objetivos do trabalho, uma vez que envolve somas de Rieemam, aqui vamos apenas utilizar o resultado para definir uma função relacionada a $A(a, b)$. Da relação anterior, se tomarmos $k=\frac{1}{a}$ podemos concluir que: $A(a, b)=A\left(1, \frac{b}{a}\right)$. Note 


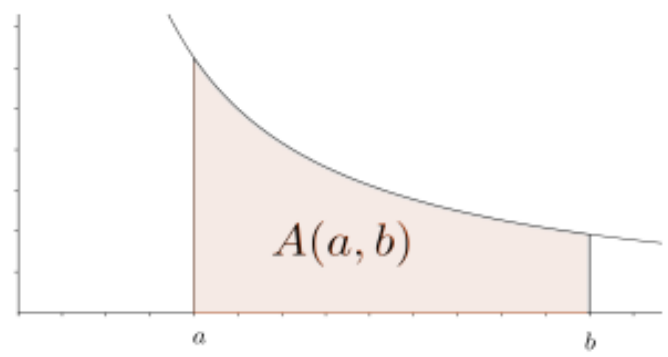

Figura 4: Ilustração

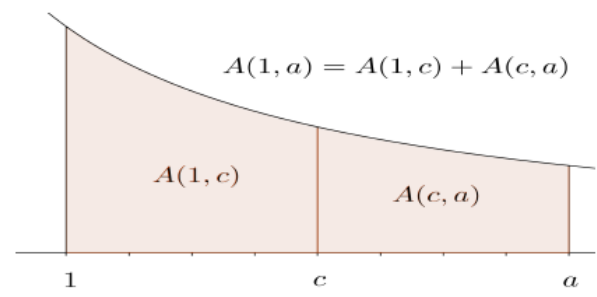

Figura 5: $\mathrm{A}(1, \mathrm{a})=\mathrm{A}(1, \mathrm{c})+\mathrm{A}(\mathrm{c}, \mathrm{a})$

que, para qualquer $c \in \mathbb{R}$, tal que $1<c<a$, temos:

$$
A(1, a)=A(1, c)+A(c, a) \text { (vide figura 5) }
$$

Temos ainda que :

$A(1, a)=A(1, c)+A(c, a) \Rightarrow A(1, a)=A(1, c)+A\left(1, \frac{a}{c}\right)^{7}$

Esse fato nos permite concluir que essa relação entre áreas "transforma"multiplicação em adição. Vamos justificar essa afirmação. Sejam $a$ e $b$ dois números reais maiores que 1 . Assim, $1<a<a . b$, portanto, pelas propriedades apresentadas nos parágrafos anteriores $A(1, a . b)=$ $A(1, a)+A(a, a \cdot b)=A(1, a)+A(1, b)$. Seja $x>1$, denotando $A(1, x)$ por $L(x)$ temos $L(a \cdot b)=L(a)+L(b)$, o que nos permite concluir que $L(x)$ é a expressão que fornece os valores funcionais de uma função logarítmica - no sentido que associa multiplicação a adição. Temos ainda que $L(1)=A(1,1)=0$ como era de se esperar.

Para $0<x<1$ convencionaremos $A(1, x)=-A(x, 1)$. Essa convenção é intuitiva, pois estamos medindo uma área no sentido oposto ao definido primeiramente. A figura 6 ilustra essa convenção.

Assim teremos

$$
\begin{gathered}
L(x)=A(1, x)=-A(x, 1)=\ldots \\
\ldots=-A\left(1, \frac{1}{x}\right)=\ldots
\end{gathered}
$$

\footnotetext{
${ }^{7}$ Na relação anterior utilizamos a propriedade $A(c, a)=A(k . c, k \cdot a)$ tomando $k=\frac{1}{c}$.
}

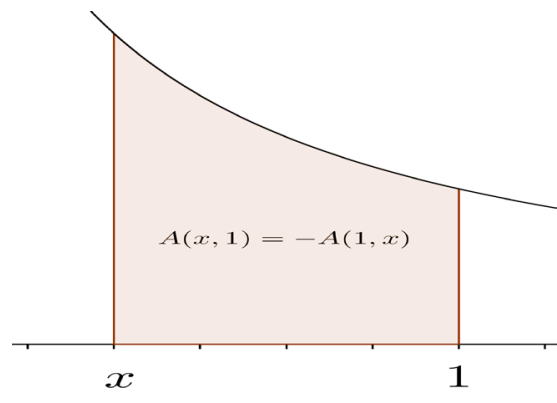

Figura 6: $L(x)$ para $0<x<1$

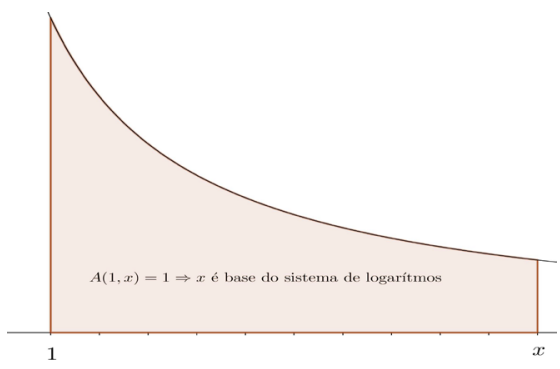

Figura 7: Base do sistema de logarítmos.

$$
\ldots=-L\left(\frac{1}{x}\right)=-L\left(x^{-1}\right) \Rightarrow L(x)=-L\left(x^{-1}\right)
$$

Com esses resultados, notamos que $L(x)$ também transforma divisões em subtrações, pois:

$$
L\left(\frac{a}{b}\right)=L\left(a \cdot b^{-1}\right)=L(a)+L\left(b^{-1}\right)=L(a)-L(b)
$$

Mas falta ainda uma questão: qual a base desse sistema de logarítmos? Conforme discutido anteriormente devemos determinar o número " $x$ " tal que $A(1, x)=$ $L(x)=1$.

Esse número é a base dos logarítmos naturais, é o número " $e$ ".

Outras bases podem ser obtidas pelo processo de áreas abaixo de outras hipérboles $\left(y=k \cdot x^{-1}\right.$ para $k \in$ $\mathbb{N}$ ). A partir dessa abordagem o aluno pode obter aprocimações do número " $e$ ", dividindo a hipérbole em retângulos e calculando suas áreas até se aproximar de 1. A próxima seção ilustra um exemplo que pode ser aplicado em sala de aula.

\section{Determinando o número " $e$ "}

Vamos calcular aproximações de $L(2)$ e $L(3)$.

\section{Intervalo (1,2)- Aproximação por excesso}




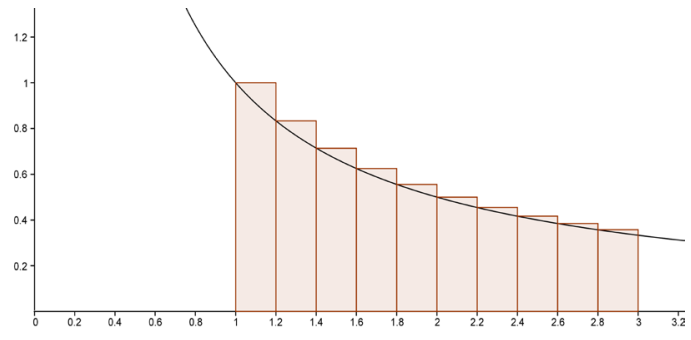

Figura 8: Aproximação por Excesso.

Vamos começar dividindo o intervalo em retângulos de mesma base, escolhemos como base 0,2 , isto é $x_{i+1}-$ $x_{i}=0,2$. Assim teremos: $x_{0}=1, x_{1}=1.2, x_{2}=1,4$, e assim por diante, até $x_{10}=3$. Como desejamos uma aproximação por excesso, tomamos a altura do retângulo como sendo $f\left(x_{i}\right)$, uma vez que $f\left(x_{i}\right)>f\left(x_{i+1}\right)$. Vale notar que quanto menor a base tomada melhor será a aproximação, no entanto, apenas queremos exemplificar, e para esse propósito a consideração feita é suficiente.

Vamos então calcular a área de cada retângulo apresentado e somá-las para assim obter a aproximação desejada. Denotaremos por $A_{i}$ a área do retângulo que tem por altura $f\left(x_{i}\right)$ e por $A_{\text {excesso } 1}$ a soma das áreas de todos os retângulos no intervalo $(1,2)$.

Assim $A_{i}=0,2 \cdot f\left(x_{i}\right)$. Então:

$$
\begin{gathered}
A_{\text {excesso } 1}=\sum_{i=0}^{4} A_{i}=0,2 \cdot \sum_{i=0}^{4} f\left(x_{i}\right) \Rightarrow \\
\ldots \Rightarrow A_{\text {excesso } 1}=0.2 \cdot\left(f\left(x_{0}\right)+f\left(x_{1}\right)+\ldots\right. \\
\left.\ldots+f\left(x_{2}\right)+f\left(x_{3}\right)+f\left(x_{4}\right)\right) \Rightarrow \ldots \\
\ldots \Rightarrow A_{\text {excesso } 1}=0.2 \cdot\left(\frac{1}{1}+\frac{1}{1,2}+\frac{1}{1,4}+\frac{1}{1,6}+\frac{1}{1,8}\right) \Rightarrow \ldots \\
\ldots A_{\text {excesso } 1}=0,7456
\end{gathered}
$$

Então temos que $L(2)=A(1,2)<A_{\text {excesso } 1}=0,7456$.

Intervalo (1,2)- Aproximação por falta A $\mathrm{O}$ processo de aproximação por falta é análogo ao anterior, ou seja, tomaremos retângulos iguais de base 0,2 calcularemos todas as áreas e somaremos. Porém, tomaremos como altura do retângulo $f\left(x_{i+1}\right)$.

Assim, designando por $A_{\text {falta } 1}$ a soma das áreas dos retângulos e por $A_{i}$ o retângulo que tem altura $f\left(x_{i+1}\right)$, teremos:

$$
\begin{aligned}
A_{\text {falta } 1} & =\sum_{i=0}^{4} A_{i}=0,2 \cdot \sum_{i=0}^{4} f\left(x_{i+1}\right) \Rightarrow \ldots \\
\ldots \Rightarrow A_{\text {falta } 1} & =0.2 \cdot\left(f\left(x_{1}\right)+f\left(x_{2}\right)+f\left(x_{3}\right)+\ldots\right. \\
\ldots & \left.+f\left(x_{4}\right)+f\left(x_{5}\right)\right) \Rightarrow \ldots
\end{aligned}
$$

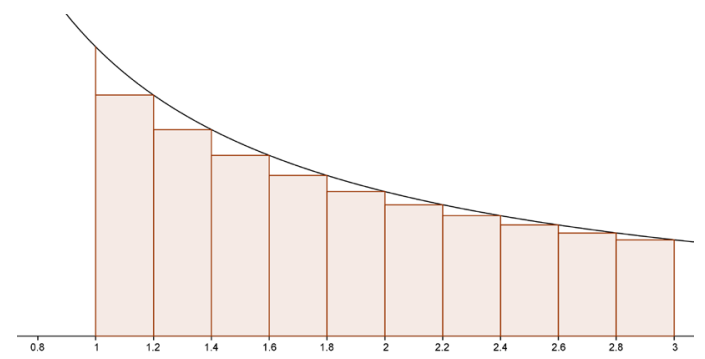

Figura 9: Aproximação por falta.

$$
\begin{gathered}
\ldots \Rightarrow A_{\text {falta } 1}=0.2 \cdot\left(\frac{1}{1,2}+\frac{1}{1,4}+\frac{1}{1,6}+\frac{1}{1,8}+\frac{1}{2}\right) \Rightarrow \ldots \\
\ldots A_{\text {falta } 1}=0,6456
\end{gathered}
$$

Então $L(2)=A(1,2)>A_{\text {falta1 }}=0,6456$. Do exposto anteriormente temos $0,6456<L(2)<0,7456$. Se diminuirmos a base tomada, teremos uma aproximação melhor, todavia os dados nos permitem concluir que 2 não é a base do nosso sistema de logarítmos, pois $L(2)<1$. Vamos agora testar o número 3.

\section{Intervalo (1,3)- Aproximação por excesso}

Para obter a aproximação por excesso de $A(1,3)$ basta somar as áreas dos 5 retângulos restantes em $A_{\text {excesso } 1}$ (vide figura 8). Assim, denotando essa aproximação por $A_{\text {excesso2 }}$ temos:

$$
\begin{gathered}
A_{\text {excesso } 2}=A_{\text {excesso } 1}+\sum_{i=5}^{9} A_{i}=\ldots \\
\ldots=A_{\text {excesso } 1}+0.2 \cdot\left(f\left(x_{5}\right)+f\left(x_{6}\right)+\ldots\right. \\
\left.\ldots+f\left(x_{7}\right)+f\left(x_{8}\right)+f\left(x_{9}\right)\right)=\ldots \\
\ldots=A_{\text {excesso } 1}+0,2 \cdot\left(\frac{1}{2}+\frac{1}{2,2}+\frac{1}{2,4}+\frac{1}{2,6}+\frac{1}{2,8}\right)= \\
=0,7456+0,4225=1,1682
\end{gathered}
$$

Então temos que $L(3)=A(1,3)<A_{\text {excesso2 }}=1,1682$

\section{Intervalo (1,3)- Aproximação por falta}

Para obter a aproximação por falta de $A(1,3)$ basta somar as áreas dos 5 retângulos restantes em $A_{\text {falta1 }}$ (vide figura 9). Assim, denotando essa aproximação por $A_{\text {falta2 }}$ temos:

$$
\begin{aligned}
A_{\text {falta } 2}= & A_{\text {falta } 1}+\sum_{i=5}^{9} A_{i}=A_{f a l t a 1}+0.2 \cdot\left(f\left(x_{6}\right)+f\left(x_{7}\right)+\ldots\right. \\
& \left.\ldots+f\left(x_{8}\right)+f\left(x_{9}\right)+f\left(x_{10}\right)\right) \Rightarrow \ldots \\
A_{\text {excesso } 2}= & A_{\text {excesso } 1}+0,2 \cdot\left(\frac{1}{2,2}+\frac{1}{2,4}+\frac{1}{2,6}+\frac{1}{2,8}+\frac{1}{3}\right) \Rightarrow \ldots \\
& A_{\text {falta } 2}=0,6456+0,3892=1,0348
\end{aligned}
$$


Então temos que $L(3)=A(1,3)>A_{\text {falta } 2}=1,0348$. Temos então que $1,0348<L(3)<1,1682$, logo podemos concluir que $L(3)>1$, sendo assim, 3 também não é a base do nosso sistema de logarítmos.

Os cálculos anteriores podem ser repetidos para outros intervalos até termos a aproximação desejada, mas já é possível notar que $2<e<3$, pois $L(2)<1<L(3)$.

\section{Disposições Finais}

O presente trabalho foi produzido como intuito de servir de inspiração para o professor na elaboração de suas aulas. A discussão através do contexto histórico permite uma melhor compreensão das propriedades dos logarítmos e da natureza do número " $e$ ".

Ao enfatizamos diversas vezes que os logarítmos foram criados para "transformar multiplicação em adição, sugerimos implicitamente que o professor inicie definindo logarítmos dessa forma. Acreditamos que, assim, a apresentação desse conteúdo no ensino básico será facilitada, uma vez que, essa abordagem torna desnecessária um conjunto de demonstrações ou justicativas que pouco contribuem para a assimilação do conteúdo. Além disso, a ampla utilização da base 10 no ensino básico encontra respaldo e uma maior compreensão com essa abordagem, perdendo assim o caráter de escolha aleatória.

Embora o surgimento do número " $e$ " tenha sido notado primeiramente em aplicações financeiras, uma das maiores aplicações na matemática é sua utilização como base de um sistema de logarítmos. Dessa forma acreditamos ser de extrema importância justificar sua utilização como base de um sistema de logarítmos, humanizando, assim, a construção do conhecimento matemático.

Portanto, com este artigo, pretendemos fornecer material aos professores da rede básica e assim nos juntarmos aos profissionais que acredtam que a história da ciência e da matemática é um agente motivador e facilitador da aprendizagem de um conceito.

\section{Referências}

CAJORI. F. Uma História da Matemática. ${ }^{\mathrm{a}}$ ed. Rio de Janeiro: Ciência Moderna, 2007.

EVES.H. Introdução a História da Matemática., $5^{\mathrm{a}}$ ed. Campinas: Unicamp, 2010.

LIMA.E.L. Logarítmos. $5^{\text {a }}$ ed. Rio de Janeiro: SBM, 2013 (Coleção do Professor de Matemática).

LIMA. E.L.Meu Professor de Matemática. $6^{\mathrm{a}}$ ed. Rio de Janeiro: SBM,2012. (Coleção do Professor de Matemática).

LIMA. E. L.; CARVALHO P.C.P ; WAGNER. E; CARVALHO. A.C. A Matemática do Ensino Médio, $5^{\mathrm{a}} \mathrm{ed}$.
Rio de Janeiro: SBM,2000. Volume 1. (Coleção do Professor de Matemática).

MAOR. E. e: a história de um número. $7^{a}$ ed. Rio de Janeiro: RECORD, 2012.

REIS.S.P. Logarítmos: uma proposta de ensino. 2014. 53f. Dissertação (Mestre em Matemática) - Instituto de biociências, letras e ciências exatas, Universidade Estadual Paulista, São José do Rio Preto,SP, 2014.

ROQUE.T, CARVALHO. J.B.P. Tópicos de História da Matemática. $1^{\text {a }}$ ed. Rio de Janeiro: SBM, 2012.

STEWART. I. Equações que Mudaram o Mundo. $3^{\text {a }}$ ed. Rio de Janeiro: ZAHAR, 2010. 